\title{
Organisations islamiques nigérianes en Côte d'Ivoire. Les trajectoires concurrentielles de NASFAT et NAMFAT
}

Issouf Binaté et Yaya Dao

\section{OpenEdition}

\section{Journals}

Édition électronique

URL : http://journals.openedition.org/anthropodev/831

DOI : 10.4000/anthropodev.831

ISSN : 2553-1719

Éditeur

Presses universitaires de Louvain

\section{Édition imprimée}

Date de publication : 1 décembre 2019

Pagination : 87-105

ISBN : 978-2-87558-940-8

ISSN : 2276-2019

\section{Référence électronique}

Issouf Binaté et Yaya Dao, « Organisations islamiques nigérianes en Côte d'Ivoire. Les trajectoires concurrentielles de NASFAT et NAMFAT», Anthropologie \& développement [En ligne], 50 | 2019, mis en ligne le 19 décembre 2020, consulté le 24 janvier 2021. URL : http://journals.openedition.org/ anthropodev/831; DOI : https://doi.org/10.4000/anthropodev.831

La revue Anthropologie \& développement est mise à disposition selon les termes de la Licence Creative Commons Attribution 4.0 International. 


\title{
Organisations islamiques nigérianes en Côte d'Ivoire
}

\author{
Les trajectoires concurrentielles de NASFAT et NAMFAT
}

\author{
Issouf Binaté ${ }^{1}$, Yaya Dao ${ }^{2}$
}

Cet article est une étude de la dynamique islamique de la communauté yorouba en Côte d'Ivoire à travers les activités de deux associations, NASFAT et NAMFAT. La première est la représentation d'une organisation éponyme créée au Nigéria en 1995, et la seconde une initiative suscitée en territoire ivoirien en 2005. Leur présence dans la sphère associative de ce pays relève de I'œuvre personnelle d'Abdou Rahman Olota qui, de retour d'un séjour à Lagos, importa l'expérience de l'entreprise religieuse de NASFAT à Abidjan où il résidait. En dépit de la naissance de NAMFAT à la suite d'une crise à NASFAT, les deux associations au fonctionnement similaire, dans une démarche d'entreprise cosmopolite, vont constituer un cadre de socialisation des Nigérians musulmans. Ce projet passera par la réalisation des activités religieuses et d'utilité publique. Dans un contexte de visibilité accrue de la religion musulmane en Côte d'Ivoire dans l'espace public ces dernières décennies, cet article analyse l'itinéraire de ces deux organisations transnationales à travers leur mode d'organisation et d'essaimage du terrain religieux, et leur implication dans les services d'utilité publique.

This article explores the Islamic dynamics of the Yoruba community in Ivory Coast drawing on the activities of two associations operating on Ivoirian soil, NASFAT and NAMFAT. The first is the counterpart of the eponymous organization created in Nigeria in 1995. The second was born in 2005, in Ivorian territory. Both are linked to the personal work of Adeniran Ramon who imported Lagos-based NASFAT to Abidjan where he was living. Despite internal dissension which led to the creation NAMFAT, a distinct religious-based organization, both associations have operated rather similarly and by promoting cosmopolitan values, both have played the role of creating a space for the socialization of Nigerian Muslims. This has involved the organization of religious activities and actions promoting common goods. In a context of increased visibility in the public space of the Muslim religion in Ivory Coast, in recent decades, this article explores the patterns of these two transnational organizations, through their organization model, their use of the religious terrain, and their involvement in common goods services.

\footnotetext{
1 Chercheur, département d'histoire, université Alassane Ouattara, Côte d'Ivoire ; binateissouf1981@yahoo.fr

${ }^{2}$ Doctorant, département d’histoire, université Alassane Ouattara, Côte d'Ivoire ; daoyaya007@gmail.com
} 


\section{Introduction}

L'expression Côte d'Ivoire, terre de convergence et d'accueil, titre d'une parution de Simon-Pierre Ekanza (2006), est une assertion qui sied à ce pays ouest-africain aux fortes potentialités économiques, en dépit des controverses et des conflits identitaires survenus au cours de la décennie 1990 (Le Pape et Vidal, 2002 ; Akindès, 2004). Une abondante production scientifique de chercheurs africanistes témoigne de cette dynamique de migration et de ce modèle d'intégration des peuples (Mandé, 2012 ; Cissé, 2013). En 1998, les résultats du Recensement général de la population et de l'habitat (RGPH) indiquaient près de $26 \%$ d'étrangers au nombre desquels on notait aussi bien des personnes en migration de travail que des entrepreneurs religieux dont, certains, organisés au sein des représentations d'associations transnationales (Fourchard et al., 2005).

Sur cette terre de brassage socioculturel, où l'islam a acquis une visibilité importante pendant la seconde moitié du XX siècle (Kaba, 1974; Miran, 2006), ce type de mouvement d'entrepreneurs religieux fit son entrée sur la scène publique avec la présence de la mission islamique Ahmadiyya dès 1961 - soit un an après l'accession du pays à l'indépendance (Yacoob, 1983). L'ouverture de l'université islamique de Médine la même année et sa politique éducative en direction des pays subsahariens (Galilou, 2003 ; Binaté et al., 2019) ont apporté une dynamique - incluant des activités de témoignage de solidarité aux musulmans nécessiteux - à cette mobilité naissante de personnes entre la Côte d'Ivoire et le monde arabo-musulman (Schulze, 1993 ; Miran, 2006 ; Binaté, 2016). Cependant, dans un contexte de méfiance de l'État ivoirien vis-à-vis de l'islam, eu égard aux mouvements subversifs dans le Moyen-Orient et l'Afrique du Nord, ces rapports ont évolué en pointillé jusqu'à l'avènement du pluralisme politique au début des années 1990.

Cette décennie d'ouverture politique, marquée par la fin du régime de parti unique, a été favorable à la promotion des libertés individuelles et notamment de la liberté d'association (Loucou, 1992). Les musulmans profitèrent de cette opportunité pour donner de l'impulsion et de la visibilité sociale à leurs entreprises religieuses. Même si ce changement intervenu ne consacra pas dans l'immédiat le rétablissement de relations harmonieuses entre le monde arabo-musulman et la Côte d'Ivoire, il a eu le mérite de contribuer à la reconnaissance officielle de quelques associations islamiques existantes (Conseil supérieur des imams, Ligue islamique des prédicateurs en Côte d'lvoire et Association des jeunes musulmans de Côte d'Ivoire) ${ }^{3}$, à la création de nouvelles organisations (telles que le Conseil national islamique ${ }^{4}$ ) et à l'installation de sections de

\footnotetext{
${ }^{3}$ Le Conseil supérieur des imams (COSIM) et la Ligue islamique des prédicateurs en Côte d'Ivoire (LIPCI) sont nés en 1988 et l'Association des jeunes musulmans de Côte d'Ivoire (AJMCI) en 1989. Ils obtinrent leur agrément au début de la décennie 1990.

${ }^{4}$ Le Conseil national islamique (CNI) vit le jour en 1993.
} 
structures transnationales dont la Fondation Saar ${ }^{5}$ et NASFAT (Nasrul-lahi-l-Fatih) - et plus tard NAMFAT (Nasrul-Minal-lahiwa Fatihu) ${ }^{6}$, I'un de ses démembrements.

La présente étude porte sur ces deux dernières organisations conduites par des musulmans originaires du Nigéria. En effet, on ignore encore avec précision la date de l'arrivée des premières communautés de ce pays établies en Côte d'Ivoire. Toutefois, des études indiquent que les crises politiques, notamment la guerre du Biafra (1967-1970), avaient conduit des cohortes de Nigérians à trouver refuge en Côte d'Ivoire au début des années 1970 (Boutet, 1992 ; Clergerie, 1994). Près de trois décennies plus tard, cette population - composée en majorité des ethnies yoruba, igbo et haoussa - était estimée à 71355 dont 48933 musulmans ${ }^{7}$, exerçant essentiellement dans le secteur du commerce. Installée en dehors de sa terre d'origine, la composante musulmane de cette communauté allait, dans un souci de recherche d'un cadre formel de socialisation de ses membres ${ }^{8}$, participer à la mise en place de NASFAT puis de NAMFAT, à Abidjan, respectivement en 2002 et 2005. Avec à leur actif la réalisation de plusieurs projets de développement communautaire et des célébrations de festivités religieuses, ces deux organisations comptent des milliers de membres résidant aussi bien à Abidjan que dans des villes de l'intérieur du pays.

L'objectif de cette étude est d'analyser le parcours d'organisations transnationales ouest-africaines en Côte d'Ivoire, à travers des activités mises au service de la socialisation de communautés établies en dehors de leur terre d'origine. L'approche anthropologique et historique utilisée repose sur des données empiriques collectées, entre 2014 et 2018, dans le cadre de deux projets distincts: l'un, une étude postdoctorale et, l'autre, un mémoire de master sur les organisations confessionnelles islamiques en Côte d'Ivoire (Binaté, 2016, 2018 ; Dao, 2016). Cette méthodologie a porté essentiellement sur des enquêtes de terrain menées auprès de plus d'une dizaine de membres de ces associations (essentiellement des commerçants), ainsi que des professionnels du fait religieux à Abidjan - où elles siègent - et dans d'autres localités du pays. L'analyse, qui a découlé du croisement des données collectées, a permis de décrire les trajectoires de ces organisations transnationales, leurs stratégies de mobilisation et d'occupation de l'espace religieux. Aussi, une attention a-t-elle été portée sur la dynamique que le transfert des savoirs religieux de NASFAT a impulsée à la communauté nigériane de ce pays d'accueil.

\footnotetext{
${ }^{5}$ La Fondation Saar était une structure panislamique basée aux États-Unis. La section ivoirienne a été agréée en 1990, soit trois ans après son installation (Miran, 2006 : 356-357).

${ }^{6}$ Nasrul-lahi-I-Fatih et Nasrul-Minal-lahiwa Fatihu sont des expressions en langue arabe signifiant respectivement " II n'y a d'aide sauf Allah » et "Secours émanant d'Allah et victoire ".

${ }^{7}$ Selon le Recensement général de la population et de l'habitat (RGHP) de 1998.

${ }^{8}$ Ce processus vise l'éducation des générations d'émigrés musulmans aux normes et valeurs culturelles yorouba, et l'intégration de nouveaux venus du Nigéria dans les circuits socio-économiques du pays d'accueil.
} 


\section{NASFAT et NAMFAT : acteurs et parcours de deux organisations islamiques transnationales}

L'émergence des organisations islamiques dans la sphère publique ivoirienne est intervenue avec le décloisonnement de la vie politique en 1990. Cette dynamique, soutenue par d'importantes activités religieuses (conférences, participations aux débats d'intérêt national, célébrations de festivités islamiques), a préparé le terrain à l'installation de la section NASFAT Côte d'Ivoire.

Entreprise religieuse transnationale selon le modèle d'organisations aux pratiques transcendant les frontières étatiques (Mary et Fourchard, 2005 : 10-13), NASFAT est une association islamique nigériane née le 5 mars 1995 à Lagos, à l'initiative de dix intellectuels musulmans de l'ethnie yorouba dans un contexte de compétition dans l'espace religieux marqué par une vitalité des mouvements néo-pentecôtistes (Peel, 2011 : 43; Murtala, 2017: 141). Suivant une tradition soufi de groupes de prières conduits parfois en dehors des cadres et des heures des cultes canoniques, ce projet de jeunes élites est parti des rencontres dominicales organisées chez Abdul Lateef Olasupo (Adetona, 2012). Ses fondateurs ont fait des études universitaires générales, et ont des profils socioprofessionnels différents. Seul I'un d'eux, Abdullah Akinbode, fils d'un imam à Ibadan, avait fait des études religieuses à l'Ahmadiyya Grammar School où il obtint une licence en études islamiques, puis un master en relations internationales et diplomatie à l'université de Lagos (Soares, 2009 : 184).

L'initiative de NASFAT avait l'ambition de soustraire les jeunes musulmans de l'influence des missions néo-pentecôtistes en les invitant à des sessions de prières (Asalatu) chaque dimanche entre $8 \mathrm{~h} 30$ et $12 \mathrm{~h} 30$, et à des veillées nocturnes (Tahajjud) de minuit à l'aube (Peel, 2011 : 44). Avec le succès de ces activités élargies aux questions du bien-être social à la base de l'effervescence autour des organisations néo-pentecôtistes, le nombre de participants s'est accru significativement et le mouvement a connu un essor fulgurant à Lagos, avant d'attirer des adhérents estimés à plus d'un million organisés au sein de 180 sections à travers le pays. Aussi, compte tenu de la mobilité des Nigérians dans l'espace sous-régional ouest-africain, l'association a réussi à s'installer au-delà des frontières de son pays d'origine, principalement en Côte d'Ivoire où une communauté yorouba existe depuis des décennies.

Originaires principalement d'Ejigbo et d'Oyo, villes situées dans l'État d'Osun dans le Sud-Ouest du Nigéria, les premiers Yorouba s'installèrent en Côte d'Ivoire avant le début de la seconde guerre mondiale (Adesina et Adebayo, 2009 : 139). Ils résidèrent dans les quartiers de Treichville et surtout d'Adjamé. C'est d'ailleurs le quartier Adjamé qui a abrité leur première mosquée construite par Mankandju Ôla dans le secteur de l'actuel SaintMichel - baptisé Temi-di-ire (littéralement "J'ai atteint mon objectif» en langue yorouba) - dans les années 1940. Ce fut d'abord autour de ce lieu de culte que la

\footnotetext{
${ }^{9}$ L'expression Temi-di-ire a été inspirée du succès dans les affaires des premiers Yorouba installés dans ce
} quartier d'Adjamé Saint-Michel qu'ils considèrent comme une terre bénie. 
communauté yorouba, notamment la composante musulmane (ou Egbe muslimi en langue yorouba), réussit à se construire progressivement. Au début de la décennie 1970, elle parvint à installer la section ivoirienne de l'Ansar-Ud-Deen Society of Nigeria ${ }^{10}$ organisation créée en 1923 en milieu yorouba à Lagos (Reichmuth, 1996). Mais bien qu'ayant obtenu de nombreux adeptes à Abidjan, l'association évolua dans l'informalité sur le plan administratif. NASFAT, récemment implantée, sera l'une des premières associations yorouba à être agréée par l'État de Côte d'Ivoire.

La création de la section ivoirienne de NASFAT est intervenue au lendemain de l'accession du pays au multipartisme qui avait vu émerger sur la scène publique les associations islamiques. Elle est l'initiative d'Abdou Rahman Olota ${ }^{11}$, un Yorouba qui, de retour à Abidjan après un séjour au Nigéria où il avait été séduit par cette organisation religieuse, entreprit de faire profiter ses compatriotes des bienfaits d'un projet similaire. Mais le projet visant plus la socialisation des Nigérians établis en Côte d'Ivoire contrairement à la mission de NASFAT Lagos qui est une réponse aux entreprises pentecôtistes -, la réalisation de cet objectif devait passer par l'adhésion des musulmans locaux dont certains étaient déjà organisés au sein de nombreuses associations. D’abord, Abdou Rahman Olota partagea son idée avec son ami et proche collaborateur, Jayeola Souleymane $^{12}$, sur les conseils duquel il noua des attaches avec les personnes représentatives de la communauté yorouba. Ce sera, d'ailleurs, cette communauté qui constituera le vivier des membres de NASFAT.

Dans cette entreprise, le soutien de deux personnes à Abdou Rahman Olota a été décisif. Il s'agit de Hasheem Titilope et de Abdouramane Ignada. Le premier, qui deviendra le président des sages de NASFAT, est un célèbre prédicateur en milieu yorouba de Côte d'Ivoire. Il jouit d'un grand respect auprès de ses compatriotes. Dans sa fonction de religieux, Hasheem Titilope avait autour de lui un nombre important de fidèles et $d^{\prime}$ admirateurs qui feront partie du premier noyau de partisans de NASFAT ${ }^{13}$. Le second, d'un profil différent, est quant à lui un intellectuel et un ancien militant de nombreuses organisations islamiques dont l'Association des élèves et étudiants musulmans de Côte $d^{\prime}$ Ivoire $(A E E M C l)$. À ce titre, il avait de l'expérience dans le domaine associatif et disposait d'un carnet d'adresses d'une grande utilité dans la mobilisation et l'adhésion de membres à la nouvelle association.

À Treichville où il résidait, Abdou Rahman Olota avait obtenu également le soutien des chefs de communautés et de leurs membres. Selon Gbadamassi Abdou Fatahi, la question

\footnotetext{
${ }^{10}$ En l'absence d'archives, il est difficile de situer avec précision la date de création de cette organisation (entretien avec Abdul Fatahi Itiladé, Abidjan, le 17/07/2018).

${ }^{11}$ Adeniran Ramon de son nom à l'état civil, Abdou Rahman Olota est né au Nigéria le 7 mai 1947. Il arrive en Côte d'Ivoire autour des années 1970. II va exercer plusieurs petits métiers avant de faire fortune dans le commerce. Il est décédé le 5 mai 2012 à Abidjan.

${ }^{12}$ Entretien avec Gbadamassi Moutiou, à Abidjan, le 22/03/2016.

${ }^{13}$ Hasheem Titilope a été le président des prédicateurs yorouba de Côte d'Ivoire. À ce titre, il officiait pour les prières du vendredi à la mosquée Ramos de Treichville de l'avenue 21, rue 45.
} 
de la filiation entre les Yorouba a joué un rôle décisif dans la captation de membres. En général, ces ressortissants du Nigéria vivent en communauté sur des considérations parentales. Cette introversion s'explique par le fait que les premiers Yorouba à s'installer en Côte d'Ivoire venaient presque tous de la même région et se connaissaient plus ou moins. Lorsque l'un d'eux adhère à un projet associatif, il contribue à faire venir ses pairs, même si ceux-ci militent déjà dans une autre association. Mais l'adhésion communautaire à NASFAT, Abdou Rahman Olota la devait à la fois à ces liens de parenté forts entre les Yorouba et à une "redevabilité » de ses compatriotes (Blundo, 2012) qu'il avait réussi à construire au fil des années à travers des activités de témoignage de solidarité. En effet, Abdou Rahman Olota était apprécié par ses pairs pour ses actions de philanthropie exprimées en termes de parrainage d'activités, de dons en matériel et de soutien financier. De l'avis de Gbadamassi Moutiou, le succès de cette mobilisation des Yorouba a également reposé sur une stratégie de communication :

Les gens avaient besoin de voir pour croire, donc il [Abdou Rahman Olota] est venu avec des éléments vidéo de séances de prière, cérémonies de NASFAT au Nigéria. II faisait sortir toutes ses télévisions et mettait les cassettes des activités de NASFAT; il invitait ses amis, les oulémas, des intellectuels à voir d'eux-mêmes ce que c'était NASFAT. (Gbadamassi Moutiou, Abidjan, le 22/03/2016)

À cette politique de sensibilisation de masse, Abdou Rahman Olota ajouta une campagne de proximité auprès des commerçants. À son magasin situé au grand marché de Treichville, il profitait de chaque occasion pour informer son voisinage et ses visiteurs de son projet. À cet effet, il avait accordé une attention particulière aux femmes dont le militantisme religieux avait atteint une visibilité importante ces dernières décennies (Miran, 2006; LeBlanc, 2007). Les cérémonies de baptêmes, de mariages, auxquelles Abdou Rahman Olota était convié parfois comme parrain, étaient des opportunités dont il se saisissait pour présenter NASFAT au public. Par ces procédés, il parvint ainsi à constituer un groupe de prières qui jeta les bases de l'implantation de la section ivoirienne de l'organisation.

La première session de prières de NASFAT en Côte d'Ivoire s'est tenue le dimanche 11 août 2002 au domicile d'Abdou Rahman Olota. Celui-ci en devint le premier président, mais avec un statut d'intérimaire en attendant la reconnaissance officielle du siège à Lagos. Le projet ayant pris forme et les activités se déroulant de manière régulière, les dirigeants déposèrent un dossier de demande d'agrément auprès de l'État ivoirien en 2004. Conformément à la loi $n^{\circ}$ 60-315 du 21 septembre 1960, NASFAT Côte d'Ivoire obtint son agrément du ministère de l'Intérieur en 2006, soit un an après la reconnaissance officielle de l'organisation par NASFAT Lagos au cours d'une grandiose cérémonie au palais de la culture de Treichville. Vu la solennité de l'évènement, le président mondial des missionnaires de cette structure, Alhaji Abdullahi Gbadé Akinbodé, effectua le déplacement en Côte d'Ivoire. NASFAT Côte d'Ivoire devint ainsi la première section hors du Nigéria à être reconnue officiellement par Lagos. Cependant, cette cérémonie, qui consacra la reconnaissance officielle de NASFAT Côte d'Ivoire, a contribué à la mise à l'écart d’Abdou Rahman Olota de la présidence de l'organisation. 
L'officialisation de NASFAT Côte d'Ivoire, en effet, s'est faite à l'issue d'une élection pour désigner son premier responsable. L'organisation de cette élection n'avait rien en commun avec les scrutins ordinaires. Elle était à la charge d'un comité appelé Choura, composé de six à douze membres. Cet organe avait pour mission la réception et l'analyse des dossiers de candidature. Puis, il conduisait l'évaluation des candidats au cours d'entretiens oraux sur des sujets relatifs à leurs ambitions pour l'association, avant de déclarer le nom du candidat retenu pour ce poste. Contre toute attente, Abdou Rahman Olota, qui avait porté le projet du Nigéria à Abidjan, n'a pas été reconduit à la tête de NASFAT désormais présidée par Abass Ismaël Olaoyé, soutenu par le siège de l'organisation à Lagos pour son niveau d'instruction ${ }^{14}$. Inspiré de Lagos, ce mode d'accession à la présidence, défini par des normes privilégiant des candidats au profil d'intellectuel, n'a pas tenu compte des réalités sociales des Yorouba auprès de qui Abdoul Rahman Olota s'était pourtant construit une notoriété. Bien que vaincu selon les textes statutaires, ce dernier réussit à profiter de ce capital symbolique pour créer une structure similaire dénommée NAMFAT (Nasrul-Minal-lahiwa Fatihu).

Cette rupture étant intervenue en 2005 , la nouvelle association débuta sa première session de prières le dimanche 21 août de la même année à son siège actuel à Treichville. Organisation apolitique et ouverte à tous les musulmans sans distinction d'origine, l'objectif de NAMFAT, selon ses statuts et son règlement intérieur, est d'œuvrer à l'émanation d'une société islamique et d'occuper sainement les croyants à travers des sessions hebdomadaires de prières, des conférences, des séminaires, etc. NAMFAT compte également contribuer à la promotion de l'islam par les actions sociales à l'instar de NASFAT avec qui l'association partage le même espace.

Une rivalité allait s'installer entre les deux organisations, créant un climat délétère entre leurs membres. Mais elle s'est limitée à une lutte de positionnement sans donner lieu à un conflit ouvert. D'ailleurs, Gbadamassi Moutiou, interrogé des années plus tard, a analysé cette rupture :

[...] comme un agrandissement de la communauté, [...] comme une grâce que Dieu a bien voulu faire à cette communauté de base. NASFAT, qui donne naissance à un bébé [NAMFAT] qui va aussi faire son chemin, grandir et élargir la famille. Si le père est sans enfant, sa gloire est un peu limitée. (Gbadamassi Moutiou, Abidjan, 19/09/2014)

Cette perception de la rupture entre Abdou Rahman Olota et NASFAT a conduit les deux parties à entretenir des rapports moins conflictuels qui allaient impacter leurs modes d'organisation et de fonctionnement.

\footnotetext{
${ }^{14}$ Après des études secondaires au Nigéria, Abass Ismaël Olaoyé s'installa en Côte d'Ivoire pour exercer dans le commerce. Il a dirigé l'organisation de 2005 à 2010 et fut suivi à ce poste par Abdourahamane Ignada (2010 à 2015) et Yusuf Amuda Kamorudeen (depuis 2015).
} 


\section{Structuration et fonctionnement de NASFAT et NAMFAT : une organisation similaire pour un mode commun d'essaimage de l'espace associatif}

À l'analyse de leurs parcours, il ressort que NASFAT et NAMFAT ont en commun une histoire et des acteurs unis au sein de la communauté yorouba par de forts liens de parenté. Même si, au niveau institutionnel, ces organisations diffèrent - dans la mesure où la première est dépendante du siège de NASFAT à Lagos et la seconde entièrement autonome -, elles restent proches en termes de mode de structuration. Une analyse des inscriptions sur les logos (figure 1) et des mécanismes de gestion mis en place témoigne de cette réalité.

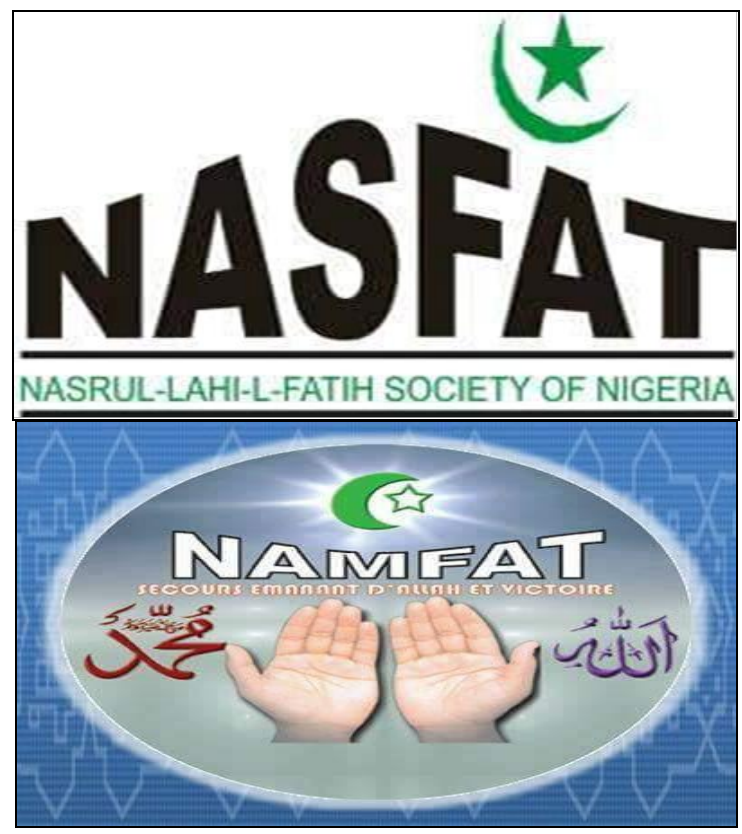

Figure 1 : Les logos de NASFAT et NAMFAT

NASFAT et NAMFAT ont, à l'exception des lettres " $S$ " et " $M$ ", les mêmes composantes signifiant littéralement en arabe "Nasrul-lahi-I-Fatih " (" II n'y a d'aide sauf Allah ») et "Nasrul-Minal-lahiwa Fatihu » ("Secours émanant d'Allah et victoire »). Une similarité qu'on retrouve au niveau des sigles disposés de manière horizontale et surmontés de la paire du croissant lunaire et de l'étoile symboles de l'islam. Cette disposition des sigles et la notion de l'assistance sociale - à travers l'utilisation des termes " aide» et "secours"- qui guide leurs actions ne sont pas loin d'une volonté de l'organisation "fille » de traduire dans la conscience collective l'idée d'une identité et d'une mission communes à celles de l'organisation " mère ".

Cette manière de faire les choses par analogie est remarquable aussi dans leur mode de gestion. L'organisation de NASFAT et celle de NAMFAT, en effet, reposent sur des organes résumés aux entités suivantes: l'assemblée générale, le conseil d'administration, le 
conseil des sages, le conseil des missionnaires, le comité exécutif des sections et le bureau exécutif. À NASFAT, ce dernier organe est à la charge du président de l'organisation. Il a un mandat de deux ans, veille à la bonne marche de l'association, exécute les résolutions prises par l'assemblée générale et approuve les programmes d'activités de tous les organes. Du côté de NAMFAT, le bureau exécutif est l'organe central. II définit l'orientation de la politique sectorielle ou des axes de développement et assure leur mise en œuvre. Ces deux organisations sont composées de plus d'une dizaine de membres.

En outre, NASFAT et NAMFAT ont, toutes deux, leur siège à Abidjan, où siège la majorité des organisations islamiques du pays. Selon les textes régissant NASFAT, le bureau d'Abidjan joue le double rôle de section-siège. Il se trouve dans la commune de Treichville, à l'avenue 21, rue 44, précisément dans un immeuble où il occupe le rez-de-chaussée et le premier étage. Concernant NAMFAT, le bureau d'où émanent les décisions de la vie de l'organisation est situé également dans la même commune. II représente le siège mondial de l'association. Ces sièges coordonnent les activités et veillent sur la bonne marche de ces structures aussi bien dans la capitale économique ivoirienne que dans les villes de l'intérieur du pays. En effet, ces entreprises, après leur lancement, n'ont pas mis beaucoup de temps pour attirer des membres en dehors d'Abidjan. Ceux-ci sont organisés au sein de structures portant les attributions de "section de NASFAT " et "section de NAMFAT » suivies du nom de la localité. Cependant, d'une localité à une autre, le processus d'installation de sections n'a pas toujours observé la même procédure.

L'expansion de NASFAT à l'intérieur du pays a suivi différentes voies, en dépit de l'existence d'une commission en charge de la coordination des sous-sections. Un extrait du discours d'Abdou Rahman Olota donne une idée du travail effectué par cet organe (NASFAT, $2014:$ 19) :

La commission chargée de la coordination des sous-sections a organisé plusieurs tournées à l'intérieur du pays pour leur rendre visite en vue de leur expliquer l'esprit dans lequel les actions se mènent à NASFAT C.I. Par leur présence sur le terrain et leur dynamisme, ils ont porté le nombre de nos sous sections du simple au double ; c'est-àdire de quatre à huit.

En procédant ainsi, les sections de Korhogo, de Soubré, etc. ont pu être installées. Mais la plus importante vague de création de sections s'est faite sans apport réel de cet organe, suivant des initiatives individuelles. Les expériences des bureaux NASFAT des villes de Bouaké et Daloa sont instructives à cet égard. L'idée d'installation de sections de cette structure de socialisation des Yorouba en migration de travail dans ces villes au centre du pays est partie du travail du réseau de partage d'informations entre les membres de cette communauté. Si, selon Atanda Tadjoudine, les Yorouba de Bouaké ont reçu l'information de l'existence de NASFAT de leurs compatriotes résidents à Abidjan, ceux de Daloa ont connu cette organisation au retour de voyage de Lagos d'Elhaji Akinsola Muhammed 
Adiatu $^{15}$. Ce dernier, une fois revenu en Côte d'Ivoire, décida avec ses amis la création de la section NASFAT de Daloa en 2003.

Ainsi, d'Abidjan, NASFAT s'est étendue à Bouaké, Daloa, Korhogo, Méagui et Soubré entre 2002 et 2013. En 2016, le nombre de sections était estimé à neuf ${ }^{16}$. Chacune d'elles bénéficie d'une autonomie tout en restant sous la supervision du siège d'Abidjan. Partant de ces dispositions, elles ont le droit de prendre des décisions jugées utiles pour la bonne marche de leurs activités. Toutefois, si l'une d'elles souhaite opérer des réformes au niveau administratif, elle doit avoir l'autorisation du siège national ou du bureau de Lagos pour des cas particuliers. Ce recours au siège international, qui se fait avec l'accord d'Abidjan, s'applique aux sections NASFAT de l'Afrique de l'Ouest. Une mesure accordant au bureau d'Abidjan une mission de plateforme incontournable au sein de ce mouvement yorouba.

À l'instar de l'ancienne organisation d'Abdou Rahman Olota, NAMFAT a des représentations dans certains pays de la sous-région ouest-africaine. Eu égard à sa particularité de structure aux mains exclusives de Yorouba et dans la logique d'entretenir les liens avec le pays d'origine, l'association s'est implantée au Nigéria (Ejigbo, Iwo, Ibadan, Edè et Lagos) et au Togo (Lomé). Cette extension du mouvement en dehors de la Côte d'Ivoire est consécutive à la mise en place des sections des villes d'Agboville, de Bouaké et de Daloa. Bien qu'il existe un secrétariat chargé des sections, ces adhésions à l'association ont plus été le fruit d'une relation de clientélisme entretenue par Abdou Rahman Olota que le résultat d'une stratégie d'essaimage de l'espace islamique établie en amont. Suivant la démarche de NASFAT, chacune de ces sections est autonome dans sa gestion administrative et tenue de rendre compte de l'évolution de ses activités annuellement au siège international à Abidjan.

En somme, après une décennie d'existence, NASFAT et NAMFAT ont réussi à se faire une place dans la communauté nigériane. En s'appuyant sur une méthode identique de travail, elles sont parvenues à faire des émules en dehors d'Abidjan où elles siègent. De plus, la première, partie de Lagos en transitant par Abidjan, a étendu ses activités à d'autres capitales ouest-africaines; quand la seconde, une initiative locale, s'est à son tour internationalisée en direction principalement du Nigéria d'où sont originaires ses membres. Cette ambition d'entreprise transnationale affichée par ces organisations ne s'est pas faite sans une politique soutenue de mobilisation de ressources.

\section{NASFAT et NAMFAT : mécanismes de mobilisation de fonds et actions d'utilité publique}

Comme cela est mentionné dans les textes portant création de ces organisations, NASFAT et NAMFAT mènent des activités apolitiques et non lucratives. Ainsi, elles

\footnotetext{
${ }^{15}$ Entretien avec Lassissi Jinan, à Daloa, le 03/04/2016.

${ }^{16}$ Entretien avec Gbadamassi Abdou Fatahi, à Abidjan, le 22/03/2018.
} 
jouissent des mêmes prérogatives que les structures associatives nationales régies par la loi $n^{\circ}$ 60-315 du 21 septembre 1960. Toutefois, leur mission, s'étendant du domaine cultuel à l'amélioration des conditions de vie de leurs membres - et des Ivoiriens par extension-, les a hissées au niveau des ONG, avec lesquelles elles bénéficient $d^{\prime}$ exonérations fiscales ${ }^{17}$ et de quelques faveurs ${ }^{18}$ de l'État. Pour assurer leur fonctionnement, les deux organisations ont élaboré des mécanismes de mobilisation de ressources financières inspirés du Nigéria (Murtala, 2017 : 174).

Cette politique de fund raising de NASFAT et NAMFAT est constituée essentiellement de la contribution financière des membres, résumée aux cotisations périodiques, à l'acquittement de la zakat et aux commissions des livraisons de biens et services. Ce mécanisme s'est construit au fil des expériences de travail. À leur installation, il a d'abord reposé sur les cotisations hebdomadaires. Les montants de ces contributions étaient laissés à la volonté des donateurs et variaient en fonction de l'évènement en projet. Aussi, dans la mesure où la majorité des adeptes de ces organisations exercent des professions libérales, la dynamique de ces participations financières reste-elle dépendante des fluctuations du marché. Ces sommes sont perçues tous les dimanches, à l'occasion des sessions de prières réunissant tous les membres. Les "secrétariats des finances " sont soumis à cette tâche, assistés par des prédicateurs mobilisateurs de la zakat (Adetona, 2012).

Dans l'islam, la zakat, troisième pilier de cette religion, est considérée comme le droit des pauvres. Elle consiste à donner le quarantième de son revenu annuel aux personnes dans le besoin. Son instauration, à cet effet, obéit à une obligation morale de réduire le fossé entre riches et pauvres (Weiss, 2000). En Côte d'Ivoire, bien que la solidarité soit inscrite dans les actions des musulmans pour le bien-être des leurs, elle reste encore non institutionnalisée, en dépit de la création de la Fondation zakat et waqf en 2010 (Binaté, 2016). À NASFAT, les missionnaires ou oulémas sont commis à la mobilisation de cette forme de dons exprimant la piété. Dans nos enquêtes de terrain, aucun chiffre ne nous a été fourni sur les montants mobilisés pendant les opérations de collecte, excepté Bouaké où la somme de 500000 francs CFA (soit 763 euros environ) est collectée régulièrement au cours des mois de Ramadan; période de choix des missionnaires pour la mobilisation de la zakat. Toutefois, rappelons qu'aucun texte de NASFAT n'oblige les membres à se plier à cette obligation, même si l'institution de la Zakat Fund demeure un élan de solidarité entre ses membres.

En plus de ces ressources mobilisées, NASFAT Côte d'Ivoire vit des retombées de sa société à responsabilité limitée dénommée FATNAS. L'idée de création de cette structure découle du modèle de Lagos (Murtala, 2017 : 175). Elle consiste à encadrer les membres du mouvement candidats au hadj ou pèlerinage annuel des musulmans en " terre sainte »

${ }^{17}$ Cette exonération est faite en application des dispositions de l'article 4 du Code général des impôts.

${ }_{18}$ L'État accompagne les organisations religieuses de son soutien à certaines occasions comme le pèlerinage à La Mecque. Cette opération consiste à prendre en charge les frais de voyage des pèlerins dont des membres de NASFAT depuis quelques années. 
du royaume d'Arabie Saoudite. Dans ce programme, FATNAS Côte d'Ivoire a d'abord joué un rôle d'auxiliaire administratif - ou de démarcheur - des services compétents de l'État (Madore et Traoré, 2018). Sous ce titre d'une sorte de cabinet de conseil, elle s'occupait d'accompagner les candidats dans la constitution des dossiers administratifs, tout en leur assurant un encadrement de proximité relatif aux rites à observer au cours de ce voyage. Pour ces services, l'organisation percevait de chaque candidat la somme de 100000 francs CFA (soit environ 153 euros). Après quelques années de travail en collaboration avec l'État, NASFAT a obtenu - par l'arrêté n 447/MEMIS/DGC - l'agrément pour l'organisation privée du hadj, à la suite d'un appel d'offres de l'État en 2013. En 2015, l'association était à sa troisième édition d'organisation du hadj et offrait deux parcours à ses pèlerins : le hadj standard et le hadj option aux prix ${ }^{19}$ respectifs de 2800000 francs CFA et 3800000 francs CFA (soit respectivement 4275 euros et 5801 euros environ). Ce dernier aussi appelé hadj VIP accorde de meilleures conditions de voyage aux pèlerins et n'excède pas 13 jours en « terre sainte ». De 2013 à 2016, FATNAS a assuré le voyage de 67 pèlerins en moyenne (tableau 1).

Tableau 1 : Récapitulatif du nombre des pèlerins de NASFAT

\begin{tabular}{|c|c|}
\hline Années du hadj & Nombre de pèlerins \\
\hline 2013 & 50 \\
\hline 2014 & 75 \\
\hline 2015 & 70 \\
\hline 2016 & 75 \\
\hline
\end{tabular}

Source : Enquêtes de terrain réalisées entre 2014 et 2018

À ce jour, les activités de FATNAS constituent la plus importante source de mobilisation de ressources financières de l'association des Yorouba. Quant à NAMFAT, elle ne dispose pas encore de mécanisme similaire. Outre la cotisation et la collecte de la zakat de ses membres, l'essentiel de sa stratégie de mobilisation de fonds se résume à la vente d'articles divers, notamment des T-shirts, des gadgets, des stylos, des polos, des écharpes, des pagnes, etc., à l'effigie de l'association.

En dehors du soutien apporté à leurs sièges respectifs - par exemple, NASFAT Côte d'Ivoire accorde $40 \%$ de ses ressources annuelles au bureau de Lagos -, l'ensemble des ressources captées par ces organisations contribue à la réalisation de leur objectif d'organisation d'utilité publique, découlant de leur conception de la $d a^{\prime} w a$ (littéralement " appel à l'islam »). En cela, ces structures transnationales s'inscrivent dans une tradition des organisations locales telle la LIPCI (Ligue islamique des prédicateurs en Côte d'Ivoire) qui, à travers sa Caravane de $d a^{\prime} w a$ (ou CADA), avait mis l'action sociale au centre de ses

\footnotetext{
${ }^{19}$ La direction générale du culte avait fixé le coût du hadj de 2015 à 2000000 francs CFA (soit 3053 euros environ) pour le hadj standard subventionné par l'État à hauteur de 800000 francs CFA (soit 1221 euros). Quant aux organisateurs privés, ils ne bénéficient d'aucun appui financier de l'État. Ce qui explique la différence de prix entre le hadj option et le hadj standard.
} 
activités de (ré)islamisation au cours de la décennie 1990. Abordant la tournure qu'avaient prises les activités islamiques à cette époque, Marie Miran expliquait que :

[...] la da'wa ne vise plus seulement à la défense et à la diffusion des dogmes religieux mais aussi à revivifier un mode de vie islamique global. Le champ d'action du prédicateur [...] n'est plus seulement la sphère spirituelle mais concerne aussi tout le domaine social. (Miran, $2000: 142$ )

Dans cette optique, ces deux organisations ont mis en œuvre des projets d'intérêt public au nombre desquels des œuvres de charité et la construction d'infrastructures. En effet, ces actions sociales intervinrent dans un contexte de crise politico-militaire qui a vu l'émergence d'organisations islamiques sur le terrain de l'humanitaire aussi bien à Abidjan qu'à l'intérieur du pays. Des ONG telles la fondation Djigui, Islam action sanitaire, Orphan's Smile, Maktab Ta Awoun, le Centre islamique Aç-habul Kahaf, le Réseau des écoles madrasas de Côte d'Ivoire, etc. se sont illustrées dans cette voie, avec la réalisation de projets dans les secteurs de l'éducation et la santé (Binaté, 2016, 2018). Suivant cette démarche, ces organisations, dans une posture d'entreprise cosmopolite (Leichtman, 2015), allaient investir les domaines séculiers. À NASFAT, les " semaines culturelles" constituent des moments dédiés à ce type d'activités. Placées sous le parrainage du $\operatorname{COSIM}^{20}$ (avec qui l'organisation yorouba entretient des rapports cordiaux), elles se présentent comme une occasion de marquage du terrain de la solidarité à travers la redistribution de fonds collectés aux nécessiteux. Des personnes membres (ou non) de l'organisation en situation difficile, la radio nationale islamique Al Bayane, l'orphelinat de Bingerville, le centre Oasis des enfants abandonnés et orphelins de Koumassi, le Centre anti-cancéreux du centre hospitalier et universitaire de Treichville, l'hôpital islamique de Daloa (ouvert le 5 mars 2018), etc. ont déjà bénéficié de cette manne financière. Une réalité reconnue par un responsable de la radio Al Bayane :

NASFAT est l'une des premières associations islamiques à faire un don important à la radio $A$ l Bayane. Ils nous font régulièrement des dons en numéraire et en matériel ; ce qui a encouragé d'autres organismes à faire de même pour la radio. (Kounadi Koné, Abidjan, le 25/02/2016)

Outre les semaines culturelles, un secrétariat des affaires sociales existe pour la gestion quotidienne des cas sociaux. Dans la section de Bouaké, la zakat collectée est offerte en don aux membres en quête de financement pour leurs projets.

On a décidé que ceux qui ont les moyens donnent leur zakat à NASFAT, même ne seraitce que la moitié, pour que ceux d'entre nous qui sont en difficulté puissent en bénéficier. Cette année, on a eu 510000 francs CFA qu'on a remis à un membre qui a été choisi par un collège de quatre personnes. Ces quatre personnes-là doivent faire ça en toute discrétion et ne doivent jamais divulguer le nom du bénéficiaire et l'argent donné n'est pas remboursable. (Atanda Tajudeen, Bouaké, le 20/05/2016)

\footnotetext{
${ }^{20}$ Le COSIM prône un islam modéré, il est actuellement la plus importante organisation islamique du pays. En plaçant ses activités sous l'autorité de cette organisation, NASFAT se démarque ouvertement des milieux wahhabites avec lesquels le COSIM est en compétition.
} 
Cette stratégie de " marketing caritatif » participe à la fidélisation des membres et à l'adhésion de nouveaux. Ainsi, elle tient une place indispensable dans la stratégie d'essaimage territorial de NAMFAT qui accorde des aides à ses membres. En 2015, à la suite d'une visite à la maison d'arrêt et de correction d'Abidjan (MACA), ses responsables ont entrepris la rénovation du décor intérieur et extérieur de la mosquée de ce centre pénitencier le plus important du pays. Sur ce chantier de réhabilitation ou de réalisation d'édifices publics, NASFAT a à son actif la construction du centre médico-social la Fontaine, ouvert le 30 janvier 2011 dans la commune d'Adjamé (Abidjan).

Cette clinique, de la même dénomination que celle de Lagos (Adetona, 2012), a été financée par NASFAT Côte d'Ivoire et délivre divers services : la consultation générale, l'odontologie, un laboratoire et une pharmacie. Depuis son inauguration, elle fonctionne sur des tarifs sociaux, suivant ses objectifs de faire du social et de faciliter la prise en charge médicale de ses membres. À certaines occasions, notamment à l'approche du jeûne de Ramadan, elle organise des séances de consultation gratuites relatives au contrôle de la tension et de la glycémie. À Méagui, au sud-ouest du pays, c'est un projet d'école primaire de trois classes qui a été réalisé. En 2016, on y comptait le niveau préscolaire, le cours préparatoire première année $(C P 1)$ et le cours préparatoire deuxième année (CP2). Contrairement aux écoles confessionnelles islamiques en général, ce projet éducatif de NASFAT a un aspect original qui réside dans son programme, avec l'anglais et l'arabe enseignés aux côtés du français. Le choix de l'arabe répond à la mission religieuse de l'entreprise et celui de l'anglais pour garder le lien avec le Nigéria, pays anglophone. À Bouaké, un projet similaire est en cours de réalisation. Sur un site de l'organisation sis au quartier Houphouët-ville, trois salles de classe, déjà sorties de terre en 2016, accueillaient plus d'une centaine d'élèves yorouba et d'autres ethnies de la ville.

Avec le succès connu à Abidjan et au-delà, ces actions sociales permirent à NASFAT et NAMFAT de faire des émules ${ }^{21}$ et surtout de se construire un capital symbolique qui sera un atout considérable pour la mission religieuse qui sous-tend leur mission. En effet, l'idée promue par ces organisations vise une participation à la promotion de l'islam par le développement d'une vie spirituelle et d'une religion portée vers la recherche du bienêtre de ses adeptes. La personne d'Abdou Rahman Olota et ses biens ont contribué à cet engagement mis au service de l'islam. À la création de NASFAT, les prières hebdomadaires, organisées les dimanches (pour détourner les jeunes des cultes festifs des églises néo-pentecôtistes), avaient lieu à son domicile, avant d'être délocalisées dans une mosquée située à Treichville. Avec la mise en place des sections communales, l'organisation décentralisa ses activités. Ainsi, les fidèles de Yopougon furent invités à faire leur session de prières à la mosquée de "Taxi gare de Siporex ", quand ceux d'Abobo et d'Adjamé se contentèrent respectivement des rencontres à la mosquée Istijaba de Gagnoa gare et la grande mosquée du boulevard Nangui Abrogoua. Avec la crise post-électorale de 2011 qui n'a pas épargné les étrangers, l'organisation, ayant vu le nombre de ses membres baisser, allait alterner les rencontres hebdomadaires entre les mosquées de

${ }^{21}$ En 2012, NASFAT avait plus de 1500 membres tandis que NAMFAT en comptait 1948 en 2014. 
Treichville et d'Adjamé. Quant à NAMFAT, chaque section organise à son lieu de culte les prières hebdomadaires.

Les séances de prières hebdomadaires s'organisent autour d'un livret - Prayerbook produit par les missionnaires de NASFAT à Lagos. Ce livret contient des bénédictions (ou $d u a$ ) tirées du Coran, des prières sur le prophète Muhammad, etc. (Adetona, 2012). Les invocations et prières sont écrites en arabe, puis traduites en anglais et en yorouba. En général, le culte commence par un culte de louange à Dieu et à Son messager, avant l'étape de la lecture du Prayerbook par les missionnaires ${ }^{22}$, soutenus en chœur par les fidèles au rythme d'une mélodie et des pas de danse. Un style de prière apparenté aux cultes des églises néo-pentecôtistes dans le sillage desquelles l'organisation fit son apparition au Nigéria (Peel, 2011 ; Murtala, 2017). Il s'ensuit un sermon sur un thème touchant au quotidien de la communauté et parfois des sessions de prières individuelles à la demande des fidèles. Si la récitation du contenu du livret se fait en arabe, les explications qui en découlent sont faites en yorouba. À Bouaké et Korhogo où la langue véhiculaire malinké reste dominante, un missionnaire est chargé de traduire les prêches aux membres ne comprenant pas le yorouba. Ces rencontres sont des occasions pour les membres de s'acquitter de leur cotisation à l'aide de cartes de paiement et de faire des offrandes de diverses natures aux missionnaires. Cependant, le choix du dimanche comme jour de culte n'est pas partagé par toutes les sections de NASFAT. C'est le cas de la section de Méagui où les membres, des commerçants en majorité, ont décidé de faire les séances hebdomadaires de prières le samedi et ont consacré le dimanche, "jour de marché », à l'écoulement de leurs produits.

En plus de ces cultes, NASFAT Côte d'Ivoire organise le premier et le troisième vendredi de chaque mois une veillée de prières appelée Tahajjud de $22 \mathrm{~h}$ à l'aube. D'ordinaire organisée pendant la dernière décade du mois de Ramadan, cette rencontre consiste en des prières et des bénédictions sur le prophète Muhammad, suivies de conférences débats sur l'islam. Ce moment de prières, outre son caractère piétiste, est un programme visant à renforcer les connaissances religieuses des jeunes (Saint-Lary et Samson, 2011 ; Sounaye, 2011) et à les détourner des night-clubs, des bars, etc. ; des espaces perçus dans l'imaginaire collectif des musulmans comme des lieux de pratiques contraires aux enseignements de l'islam. Malgré cette visée, la veillée nocturne n'est pas encore incluse au programme de $d a^{\prime} w a$ de NAMFAT.

S'appuyant sur un livret de prières constitués de sourates, d'invocations (dhikr) et de prières sur le prophète Muhammad ${ }^{23}$, NAMFAT organise ses prières hebdomadaires tous les dimanches. Celles-ci se déroulent suivant le modèle de NASFAT. Ces séances sont

\footnotetext{
${ }^{22}$ Ces religieux sont choisis sur place ou sur proposition de Lagos selon leur connaissance religieuse et leur moralité. Hasheem Titilope est le plus connu d'entre eux. Toutefois, la commission des missionnaires est dirigée par un quadragénaire, Alhaji Idris Fasasi Abolayo.

${ }^{23}$ Appelé Gbigba Adua (littéralement " recueil de doua » en yorouba), le livret de prière de NAMFAT est inspiré de celui de NASFAT. La seule différence notable entre les deux livrets est l'absence de traduction anglaise dans celui de NAMFAT. On n'y trouve que l'arabe et le yorouba.
} 
dirigées par des oulémas à qui il revient la charge de la récitation du livret en arabe et des prêches en yorouba. À l'instar de NASFAT, les membres de NAMFAT sont encouragés à mémoriser le contenu du livret et à prendre part aux activités annuelles comme les célébrations de la naissance du Prophète Muhammad ou Maouloud (expression dérivée de Mawlid an-Nabi en arabe).

Ces festivités se tiennent au cours du troisième mois du calendrier lunaire et bénéficient d'une grande attention des musulmans ouest-africains ces dernières décennies (Holder et Olivier, 2014 ; Binaté, 2017), en dépit des débats opposant encore les musulmans de différents courants sur leur légitimité (Triaud, 1979 ; Miran, 2006). Ce débat n'a pas fait l'objet d'une préoccupation à NASFAT et NAMFAT, plus proches des malékites que des groupements wahhabites. Leurs initiatives que d'aucuns qualifient d'actes mustahhab (c'est-à-dire surérogatoires ou "recommandés et non obligatoires » en langue arabe) (Peel, 2011 : 45) prennent en compte les célébrations de la naissance du prophète de l'islam dans leur programme d'activités. À ces occasions, chacune des sections organise des veillées de prêches au cours desquelles l'histoire de la religion musulmane est enseignée, avec un accent sur la vie du prophète Muhammad. Après le décès d'Abdou Rahman Olota en 2012, les nouvelles autorités de NAMFAT ont instauré des célébrations de Maouloud tournantes pour les sections d'Abidjan.

En définitive, NASFAT et NAMFAT ont réussi à étendre les activités de leurs sièges d'Abidjan aux villes de l'intérieur du pays. La réalisation de nombreux projets est à leur actif, tant dans le domaine religieux que dans le séculier. Cet essor de ces missions a reposé sur des ressources financières importantes mobilisées à partir des cotisations, des dons, des revenus des biens et services offerts par ces organisations.

\section{Conclusion}

L'utilisation du social à des fins religieuses avait été longtemps le fait des mouvements chrétiens, qui seront au cours de la décennie 1990 accompagnés par d'autres communautés religieuses. Dans le contexte de crise économique qui prévalait, les musulmans allaient mettre sur pied des ONG pour venir en aide à leurs coreligionnaires (Binaté, 2016, 2018). Dans cet élan, ils furent rejoints par Abdou Rahman Olota à I'initiative de la création de NASFAT et NAMFAT, des organisations nigérianes en Côte d'Ivoire, à partir de 2002. Si l'implantation de ces deux organisations a emprunté divers chemins, ce commerçant yorouba s'est investi personnellement et financièrement pour que celles-ci soient une réalité en territoire ivoirien. De par leur origine, elles ont réussi à mobiliser la communauté yorouba autour d'elles. Plus d'une décennie après leur implantation, elles sont en train de devenir des acteurs importants dans le milieu associatif islamique nigérian à travers une stratégie de $d a^{\prime} w a$ basée sur les activités religieuses d'une part et les œuvres sociales d'autre part.

Cependant, l'élan militant de NASFAT et NAMFAT est freiné par l'étiquette d'identité yorouba associée dans l'imaginaire populaire à ces organisations. En dehors de la communauté yorouba, les deux organisations éprouvent des difficultés à assurer leur 
cosmopolitisme bien qu'offrant des services appréciés des autorités publiques et des musulmans locaux. En effet, l'implantation de NASFAT et la création de NAMFAT ont été possibles grâce à la diaspora yorouba, représentant la quasi-totalité de leurs membres. Le yorouba s'est donc imposé comme leur langue officielle. Cette influence ethnique est perceptible à travers leurs livrets de prières en arabe, traduites uniquement dans cette langue. Malgré les manifestations festives placées sous l'autorité de la plus importante organisation islamique et les tentatives de traduction des prêches en d'autres langues lors des célébrations religieuses, ces associations ne sont pas encore parvenues à retenir de manière remarquable les membres d'autres groupes ethniques. Or il est de notoriété publique que le malinké demeure la langue principale de transmission du message islamique et le français celle du discours de l'élite musulmane en Côte d'Ivoire (GuyonMiran et Oyewolé, 2015).

\section{Bibliographie}

Adesina Y.R., Adebayo F.P., 2009, "Yoruba traders in Cote d'Ivoire: A study of the Role Migrant Settlers in the Process of Economic ", African Research Review, n 3(2), pp. 134-147.

Adetona L.M., 2012, « NASFAT : A Modern Prayer Group and its Contributions to the Propagation of Islam in Lagos ", World Journal of History and Civilization, $n^{\circ} 2(2)$, pp. 102-107.

Akindès F., 2004, Les racines de la crise militaro-politique en Côte d'Ivoire, Dakar, CODESRIA.

Binaté I., 2016, "Muslims NGOs in Côte d'Ivoire: Towards an Islamic Culture of Charity », in M.-N. LeBlanc, L. Audet-Gosselin (éds), Faith and Charity : Religion and Humanitarian Assistance in West Africa, London, Pluto Press, pp. 47-62.

Binaté I., 2017, "Les célébrations du Maouloud au nord de la Côte d'Ivoire : entre espace de réislamisation, socialisation et quête de légitimité politique ", Cahiers d'études africaines, $\mathrm{n}^{\circ} 225$, pp. 39-58.

Binaté I., 2018, "Les ONG islamiques en Côte d'Ivoire en période de conflit : acteurs et enjeux », Repères, $\mathrm{n}^{\circ} 1(1)$, pp. 193-230.

Binaté I., Ouédraogo Y., Audet-Gosselin L., 2019, « Être arabisant en Afrique francophone : regards croisés sur des élites burkinabè et ivoiriennes formées en pays arabo-musulmans ", Islam et Société au Sud du Sahara, Paris, Les indes savantes, nouvelle série, vol. 5, pp. 9-30.

Blundo G., 2012, « Le roi n'est pas un roi : les multiples redevabilités au sein de l'État postcolonial en Afrique ", in P. Haag, C. Lemieux (éds), Faire des sciences sociales. Critiquer, Paris, Éditions de l'EHESS, pp. 59-86.

Boutet R., 1992, L'effroyable guerre du Biafra, Paris, Éditions Chaka.

Cissé C., 2013, Migration et mise en valeur de la Basse Côte d'Ivoire (1920-1960), Paris, L'Harmattan.

Clergerie J.L., 1994, La crise du Biafra, thèse de doctorat d'État, université de Limoges, Presses Universitaires de France, $385 \mathrm{p}$.

Dao Y., 2016, NASFAT et NAMFAT en Côte d'Ivoire, mémoire de Master, université Alassane Ouattara, Bouaké, $178 \mathrm{p}$. 
Ekanza S.P., 2006, Côte d'Ivoire: Terre de convergence et d'accueil (XV et XIX siècle), Abidjan, CERAP.

Fourchard L., Mary A., Otayek R. (éds), 2005, Entreprises religieuses transnationales en Afrique de l'Ouest, Paris-Ibadan, Karthala-IFRA.

Galilou A., 2003, "The graduates of Islamic universities in Benin: A modern elite seeking social, religious and political recognition ", in T. Bierschenk, G. Stauth (éds), Yearbook of the sociology of islam, Islam in Africa, $n^{\circ}$ 4, pp. 129-146.

Guyon-Miran M., Oyewolé N., 2015, « Côte d'Ivoire, un Islam d'expression française à la fois tangible et restreint ", in I.R. Yassine (éd.), Histoire, monde et cultures religieuses, $n^{\circ} 16$, Paris, Karthala, pp. 141-158.

Holder G., Olivier E., 2014, « Le Maouloud de Djenné : stratégies patrimoniales de l'islam, mémoire urbaine et identité nationale ", in J. Brunet-Jailly et al. (éds), Le Mali contemporain, BamakoParis, Éditions Tombouctou - IRD Éditions, pp. 263-294.

Kaba L., 1974, The Wahhabiyya: Islamic Reform and Politics in French West Africa, Evanston, Northwestern University Press.

Le Pape M., Vidal C. (éds), 2002, Côte d'Ivoire : I'année terrible (1999-2000), Paris, Karthala.

LeBlanc M.N., 2007, "Imanya and Young Muslim Women in Côte d'Ivoire ", Anthropologica, $n^{\circ} 49(1)$, pp. 35-50.

Leichtman A.M., 2015, Shi'i cosmopolitanism in Africa: Lebanese Migration and Religious Conversion in Senegal, Bloomington, Indiana University Press.

Loucou J.N., 1992, Le multipartisme en Côte d'Ivoire, Abidjan, Neter.

Madore F., Traoré Y., 2018, "L'organisation du hadj en Côte d'Ivoire : entre facteur de cohésion et source de rivalités au sein de la communauté musulmane (1993-2010) ", Cahiers des études africaines, $\mathrm{n}^{\circ} 229$, pp. 179-208.

Mandé I. (éd.), 2012, "Les migrations ouest-africaines en Côte d’lvoire ", Migrations société, $n^{\circ} 24(144)$.

Mary A., Fourchard L., 2005, "Introduction : réveils religieux et nations missionnaires ", in L. Fourchard et al. (éds), 2005, Entreprises religieuses transnationales en Afrique de l'Ouest, ParisIbadan, Karthala-IFRA, pp. 9-18.

Miran M., 2000, "Vers un nouveau prosélytisme islamique en Côte d'Ivoire: une révolution discrète ", Autrepart, n 16, pp. 139-160.

Miran M., 2006, Islam, histoire et modernité en Côte d'Ivoire, Paris, Karthala.

Murtala I., 2017, Sensational Piety : Practices of Mediation in Christ Ambassy and NASFAT, Utrecht University, Quaestiones Infinitae, Publications of the Department of Philosophy and Religious Studies.

NASFAT, 2014, $12^{e}$ Anniversaire et Cérémonie d'investiture du Bureau Exécutif, Abidjan, Mikki Print.

Peel D.Y.J., 2011, "Un siècle d'interactions entre islam et christianisme dans l'espace yoruba », Politique africaine, $\mathrm{n}^{\circ} 123(3)$, pp. 27-50.

Reichmuth S., 1996, "Education and Growth of Religious Associations among Yoruba Muslims : The Ansar-Ud-Deen Society of Nigeria ", Journal of Religion in Africa, $n^{\circ} 26(4)$, pp. 365-405. 
Saint-Lary M., Samson F., 2011, "Pour une anthropologie des modes de réislamisation. Supports et pratiques de diffusion de l'islam en Afrique subsaharienne", Ethnographiques.org, 22, http://www.ethnographiques.org/2011/Saint-Lary-Samson (consulté le 20/10/2019).

Schulze R., 1993, "La da'wa saoudienne en Afrique de l'Ouest ", in R. Otayek (éd.), Le radicalisme islamique au sud du Sahara : Da'wa, arabisation et critique de l'Occident, Paris, Karthala, MSHA, pp. 21-35.

Soares F.B., 2009, "An Islamic social movement in contemporary West Africa: NASFAT of Nigeria », in S. Ellis, I. Van Kessel (éds), Movers and Shakers: Social Movements in Africa, Boston-Leiden, Brill, pp. 178-196.

Sounaye A., 2011, "La "discothèque" islamique: CD et DVD au cœur de la réislamisation nigérienne ", Ethnographiques.org, 22, http://www.ethnographiques.org/2011/Sounaye (consulté le 20/10/2019).

Triaud J.L., 1979, «Le mouvement réformiste en Afrique de l'Ouest dans les années 1950 », Mémoire du CEERMA, Paris, pp. 195-212.

Weiss H., 2000, "Zakat in northern Ghana: Not an institution but a goal to be achieved ", Hemisphere, $\mathrm{n}^{\circ} 15$, pp. 141-157.

Yacoob M., 1983, Ahmadiyya and urbanization : migrant women in Abidjan, African Studies Center, Boston University, $\mathrm{n}^{\circ} 75$, working paper. 